\title{
LOS MILITARES EN EL GOBIERNO LOCAL ARAGONÉS TRAS EL DECRETO DE NUEVA PLANTA
}

\author{
José Antonio MORENO NIEVES
}

Universidad de Alicante

\begin{abstract}
Resumen
En el presente artículo se analiza la presencia de militares de profesión en el gobierno local del reino de Aragón tras la implantación del nuevo sistema municipal en 1707, destacando sus relaciones con las oligarquías locales, su origen socio-económico y cursus honorum, así como la actitud de los diferentes órganos de poder ante la militarización de los gobiernos municipales.
\end{abstract}

\begin{abstract}
We are analyzed in the present article the presence of professional soldiers in the local government of the kingdom of Aragon after the installation of the new municipal system in 1707 , emphasizing their relationships to the local oligarchies, their socio-economic origins, their «cursus honorum», and the attitude of the different power organs faced with the municipal governments' militarization.
\end{abstract}

La introducción del sistema municipal castellano en tierras aragonesas a partir de 1707 originó la transformación de la elite de poder local a distintos niveles, tales como la presencia de la nobleza titulada, la desaparición de los menestrales y comerciantes, el dominio de la baja nobleza, etc; pero aquí nos queremos detener en la llegada de un nuevo colectivo a los grupos dirigentes de las ciudades y villas aragonesas: los militares profesionales.

La escasa, por no decir nula, presencia de militares profesionales en el municipio foral aragonés había estado determinada por dos cuestiones: el mayor control que ejercía el propio municipio en la elección de sus dirigentes a través del sistema de insaculación, exigiendo la residencia efectiva en el municipio, lo que era incompatible con la carrera militar activa, y, por otra parte, la exigua presencia de aragoneses en los ejércitos de la monarquía, excepto la alta nobleza, que, en cambio, tenía vetada su entrada en los ayuntamientos forales. 
Pero la situación va a cambiar a partir de 1707 por la eliminación de las dos trabas anteriormente citadas: la desaparición del régimen municipal foral y la mayor presencia de aragoneses en los cuerpos militares profesionales. Ahora bien, lo que trataremos de dilucidar es si la llegada de los militares modificó la composición de la elite local respecto a la época foral o si fue el grupo dominante el que encontró en la milicia un nuevo método para consolidar el poder. Sea como fuere, su presencia viene a apoyar a quienes tratan de ver en el siglo XVIII las precondiciones que configuraron la importancia del militarismo dentro de la vida pública española tras el derrumbe del Antiguo Régimen ${ }^{1}$.

\section{PRIMER CONTACTO ENTRE GOBIERNO LOCAL Y EJÉRCITO: LAS MILICIAS EN LA GUERRA DE SUCESIÓN}

Aunque en principio nuestro estudio se centra en los militares profesionales, no podemos dejar de lado una referencia a las milicias locales y provinciales. $Y$ ello por dos motivos: en ellas se va a producir el primer contacto duradero entre las autoridades municipales aragonesas y el ejército, y, por otra, porque fue el punto de arranque mediante el cual la elite local vio las posibilidades que la carrera militar ofrecía en la nueva coyuntura.

La formación de las milicias locales se inicia en septiembre de 1705 en lugares como Fraga, Maella, Labata, Barbastro, Zaragoza, Tarazona, Borja y Huesca, para extenderse a otros muchos en 1706; además el Reino levantó un ejército de 2.000 hombres, organizado por el Arzobispo de Zaragoza y virrey de Aragón, en el que debían cooperar las distintas localidades a razón de su población.

Más tarde, cuando se instauraron los primeros gobiernos municipales borbónicos, la fidelidad a la nueva dinastía será uno de los méritos que más se tendrán en cuenta y nada había mejor para corroborarla que poder aducir la participación en el ejército de Felipe $\mathrm{V}$, bien en los cuerpos regulares o en las milicias locales y provinciales.

Deteniéndonos ahora en este último caso, observamos la presencia de buen número de regidores que podían alegar dicho mérito para acceder al recién instaurado régimen municipal. Su existencia es, naturalmente, más numerosa en aquellas poblaciones que habían creado su propia milicia y cuyos vecinos se habían manifestado fieles a Felipe V: Fraga, Jaca o Tarazona. En el resto, los casos son más esporádicos, aunque, como veremos, se compensó con la entrada de militares profesionales.

La imbricación entre milicias y gobierno local se constata en la organización de aquellas. La formación de la milicia se encomendaba, generalmente, a la máxima autoridad municipal, el Justicia, que pasaba a ejercer el cargo de coronel de las tropas ${ }^{2}$. El resto de autoridades o elementos destacados de la localidad, miembros de la baja nobleza, recibían los grados de teniente coronel, capitán o teniente de los distintos regimientos formados.

1. GIMÉNEZ LÓPEZ, Enrique: Militares en Valencia (1707-1808). Alicante, 1990, p. 195.

2. Es el caso de los Justicias de Borja Juan Ant ${ }^{\circ}$ Mañas, de Fraga, Pedro Foradada, y de Tarazona Francisco José Alabiano. 
La labor de estas milicias se vio condicionada por la falta de cuerpos regulares en el reino de Aragón, lo que provocó que su primera actuación se concretase en la defensa de su propio territorio, o de las comarcas cercanas, al no poder enviarse tropas reales para efectuarla. En este aspecto las más significadas fueron las milicias de Tarazona, Borja y Jaca. En esta última ciudad estuvieron ayudadas por milicias francesas, provenientes de Bearn, aunque la importancia de las tropas jacetanas en la pertinaz defensa de la ciudad, que nunca pasó a manos austracistas, se demuestra en que llegaron a formarse hasta diez compañías. Su actividad estuvo determinada por los dos asedios sufridos, teniendo que efectuar salidas en varias ocasiones a la comarca en busca de avituallamiento. Las milicias de Tarazona y Borja participaron activamente en la defensa de esta zona fronteriza con Navarra y Castilla, aunque no lograron evitar la entrada de las tropas austracistas, apoyadas por la cercana localidad de Magallón, que se significó activamente a favor del Archiduque.

La pérdida del Reino a manos austracistas en 1706 y 1710 provocó que algunas de estas tropas tuvieran que incorporarse a los ejércitos regulares y se retiraran a las zonas donde éstas estaban actuando, fuera de los límites de Aragón. Una milicia de la Comunidad de Teruel, levantada en 1705, intentó auxiliar al Regimiento de Pozoblanco en Valencia, pero su disolución por la entrada austracista, lo evitó. Sin embargo, el Procurador General de la Comunidad, José Marco Toyuela, logró alistar un pequeño número de fieles borbónicos, que se incorporaron a las tropas del Presidente del Consejo de Aragón. De la misma manera, la milicia de Tauste, al mando del capitán Francisco de Ribas, más tarde regidor tras la ocupación de la villa, pasó al reino de Navarra, acantonándose en Fustiñana.

Otro grupo de aragoneses que más tarde formarán parte de los gobiernos locales estuvo compuesto por los que engrosaron los ejércitos regulares en calidad de oficiales, atendiendo a su rango nobiliario.

La mayoría de ellos limitaron su participación a las campañas aragonesas, pero el curso de la guerra les llevó, en algunos casos, fuera del Reino. Juan Pérez Toyuela, elegido más tarde para el primer ayuntamiento borbónico de Albarracín, se incorporó al Regimiento de don José Ibáñez en las campañas del reino de Valencia. Otros pasaron a Castilla, fundamentalmente tras la ocupación austracista de 1710, participando en las batallas de Brihuega y Villaviciosa, como Fernando Navarro de Antillón, regidor de Calatayud desde 1712, que sirvió en el Regimiento del marqués de Pozoblanco.

Incluso familias enteras manifestaron su fidelidad alistándose en las tropas regulares, como sucedió en Benabarre con los Jiménez de Bagués ${ }^{3}$. Tras el intento de defensa de la localidad, José Jiménez de Bagués fue hecho prisionero y enviado a Barcelona, de donde salió tras el pago de un rescate de 1.000 doblones; aunque volvió a la localidad, fue de nuevo hecho prisionero y enviado a Lérida. Mientras, su padre había salido a Zaragoza para tratar con el Gobernador del Reino la defensa de la co-

3. Todos ellos miembros de la oligarquía local: el padre de Pedro Miguel había sido teniente del Justicia del Condado de Ribagorza entre 1680 y 1705 y más tarde regidor del primer ayuntamiento borbónico; su hijo Alejo fue regidor y José lo intentó, aunque la presencia de su padre, primero, y su hermano, más tarde, se lo impidió, pero ocupó otros cargos menores. 
marca de Ribagorza, comprando armas y caballos para una compañía que había formado otro de sus hijos, Alejo. José Jiménez de Bagués participó, a continuación, activamente en la recuperación de Ribagorza con tropas de húsares del Marqués de Saluzzo, aunque con un éxito momentáneo.

Tanto los que sirvieron en las milicias como los que lo hicieron en los cuerpos regulares volvieron a sus ocupaciones anteriores a la guerra y a sus puestos de responsabilidad en la administración local o provincial ${ }^{4}$, excepto aquellos que perdieron la vida como Gregorio Royo de Bernabé, regidor de Teruel, muerto por los miqueletes en 1708 a los pocos meses de su nombramiento, y los que continuaron en el ejército como profesionales.

\section{LA PRESENCIA DE MILITARES EN LOS AYUNTAMIENTOS DEL XVIII}

La existencia de un grupo de militares profesionales en los gobiernos locales del reino de Aragón fue uno de los cambios ligados a las modificaciones institucionales nacidas de los Decretos de Nueva Planta.

La importancia de los militares en el conjunto del siglo XVIII no fue en términos generales todo lo destacada que las circunstancias en que se produjo podía hacer pensar. Sin embargo, esta magnitud varía entre los distintos municipios, destacando su presencia en la capital del Reino.

Cuadro 1: Distribución geográfica de los regidores militares (1707-1788)

\begin{tabular}{|l|r|r|l|c|c|}
\hline MUNICIPIO & \multicolumn{2}{|c|}{ REGIDORES } & \multicolumn{2}{c|}{ MUNICIPIO } & \multicolumn{2}{c|}{$\begin{array}{c}\text { REGIDORES } \\
\mathbf{n}^{\mathbf{0}}\end{array}$} & \multicolumn{1}{c|}{$\boldsymbol{\%}$} \\
\hline ALBARRACÍN & 5 & 17,2 & HUESCA & 9 & 17,3 \\
\hline ALCAÑIZ & 7 & 12,7 & JACA & 0 & 0 \\
\hline BARBASTRO & 1 & 2,9 & MAGALLÓN & 0 & 0 \\
\hline BENABARRE & 5 & 21,7 & SÁDABA & 1 & 3,5 \\
\hline BORJA & 2 & 6,8 & SOS & 3 & 8,5 \\
\hline CALATAYUD & 6 & 10,9 & TARAZONA & 4 & 10,8 \\
\hline DAROCA & 7 & 17,0 & TAUSTE & 2 & 8,0 \\
\hline EJEA & 1 & 3,0 & TERUEL & 5 & 10,6 \\
\hline FRAGA & 7 & 5,9 & UNCASTILLO & 0 & 0 \\
\hline ZARAGOZA & 33 & 27,0 & ARAGÓN & $\mathbf{9 8}$ & $\mathbf{1 2 , 8}$ \\
\hline
\end{tabular}

4. Francisco José Alabiano que fue nombrado Corregidor de Carrión y Sahagún en 1708, para pasar en 1711 al puesto de Superintendente General de Rentas Reales de Palencia, volvió en 1715 a su puesto de regidor de Tarazona. 
La distribución geográfica de los regidores militares dependía de diversos factores; sin duda su fuerte presencia en Zaragoza se debe al propio hecho de la capitalidad del Reino, donde muchos se instalaban tras su retiro, adscritos a su plaza militar, pero también a la composición social del ayuntamiento, el único donde existía una presencia destacada de la alta nobleza mediante la reserva del $25 \%$ de sus plazas. Más difícil es la explicación de su alta proporción en Benabarre, aunque el análisis de su perfil nos demuestra que la mayoría de los regidores militares ocuparon su plaza en los primeros veinte años del periodo, tratándose de sujetos que participaron en la Guerra de Sucesión y más tarde continuaron en el ejército como profesionales. Por contra, parece extraña la ausencia de regidores militares en la ciudad de Jaca, plaza de armas, pero quizá las difíciles relaciones con la población militar del castillo y particularmente con su corregidor (a su vez gobernador de él) provocaron las reticencias de la oligarquía local a abrir el paso a este grupo profesional.

Cuadro 2: Distribución cronológica de la entrada de regidores militares ${ }^{5}$

\begin{tabular}{|l|l|l|l|l|}
\hline $1707-1728$ & $1729-1748$ & $1749-1768$ & $1769-1788$ & $1789-1808$ \\
\hline $23(8,6 \%)$ & $19(12,7 \%)$ & $20(16,2 \%)$ & $13(14,4 \%)$ & $18(17,4 \%)$ \\
\hline
\end{tabular}

Por lo que respecta a su distribución cronológica, la tendencia que se observa es de un crecimiento constante a lo largo del periodo 1708-1808. No es extraño que a comienzos de la época borbónica la presencia sea escasa ya que al tratarse de militares profesionales siguieron ocupando su empleo militar hasta el retiro, lo que se produjo años más tarde, para entonces ocupar plazas de regidores. Esta tendencia ascendente se vio cortada en el periodo 1768-1789, lo cual corroboraría la momentánea victoria del civilismo sobre el militarismo durante el auge del reformismo borbónico. Sin embargo, la política exterior de finales de siglo volvió a facilitar el acceso de militares profesionales a los consistorios aragoneses.

\section{I. Características socio-profesionales}

a) El dominio de la nobleza

La mayor parte de los regidores militares pertenecían al estamento nobiliario. Este hecho estaba fundamentado por dos cuestiones: la preponderancia de dicho estamento dentro de los mandos del ejército del siglo XVIII y el perfil nobiliario de la oligarquía local aragonesa. Por ello, es necesario realizar otro tipo de diferenciación respecto a sus rasgos sociales para conocer la cohesión de este grupo dirigente.

En primer lugar, dentro de la alta nobleza se inscribían 22 regidores $(23,5 \%$ del total), si bien concentrados en una sola población, Zaragoza, como consecuencia de que en ella residía buena parte de la misma. Tan sólo en Alcañiz tienen también cierta presencia, aunque prácticamente reducidos a una sola familia, los marqueses de Santa Coloma. El resto pertenecían a la baja nobleza, aunque los términos con que aparecen

5. Los porcentajes están referidos al total de regidores nombrados en el reino de Aragón por el Rey o la Cámara en cada periodo. 
en las fuentes son muy variados: los hay hidalgos, infanzones (o ambos términos indistintamente), caballeros (algunos con la catalogación simultánea de hidalgos o infanzones) y otros más difusos como el de «familia de primera calidad». Un pequeño grupo, por último, formaban parte de ese grupo intermedio que suponían los llamados ciudadanos, que, aunque no pertenecieran a la baja nobleza, se habían asimilado a ella. Incluso dos regidores únicamente podían alegar como origen social distinguido su limpieza de sangre y el carácter de labradores honrados de sus antecesores.

La configuración social expuesta asemeja este grupo dirigente al resto de los regidores aragoneses, pero la cuestión que se nos plantea a continuación es si su acceso supuso una quiebra en el grupo oligárquico o si se trataba de una nueva profesión ejercida por sus miembros. El análisis del origen geográfico, sus antecedentes y enlaces familiares apuntan hacia la segunda hipótesis, ya que tan sólo 29 de estos regidores (el 30,85\% del total) no podemos incluirlos entre la oligarquía.

\section{b) Oligarquía militar y militares hacia la oligarquía local}

La hipótesis de que este grupo dirigente pertenecía ya a la oligarquía local se corrobora con el hecho de que la mayoría de ellos tenían uno o varios antecedentes directos en el gobierno local aragonés, bien en época foral o bien tras la aparición del gobierno borbónico. El grado de dominio de estas familias abarcaba, además, otros ámbitos del poder local; tal es el caso de Juan Antonio Millán, que llegó a ser regidor de Alcañiz en 1785, tras su retiro como cadete de infantería, cuyo padre y abuelo habían sido ya regidores. Asimismo, su primohermano había ocupado el cargo de Síndico, y su tío el de Recaudador de Rentas y Administrador de los molinos municipales y Mayordomo de Propios.

Esta oligarquía local, que adquiere una nueva dimensión con la participación en el ejército, ya estaba presente también en otras instancias del poder, más allá de su propio municipio. Así, algunos tenían antecedentes familiares que habían intervenido en la administración provincial como corregidores o alcaldes mayores. Incluso algunos podían blasonar de familiares en puestos más destacados como la Audiencia aragonesa o los Consejos, tanto en época foral como en la borbónica. Entre los regidores de Zaragoza, el padre de Antonio Blanco, marqués de Villasegura, había sido consejero criminal, oidor y regente de la Audiencia foral y consejero del de Aragón; por su parte Rafael Franco Fernández de Moros era sobrino de Diego Franco de Villalba que fue Alcalde del crimen y oidor de la Audiencia borbónica desde su creación y consejero honorífico de Hacienda. Pero no sólo los regidores de la capital tenían buenos enlaces, como demuestra la familia de Fernando París Íñiguez de Abarca, regidor de Sos, cuyo tío, Diego Íñiguez de Abarca, había sido ministro del Consejo de Navarra, Regente del Consejo de Italia, Gran Canciller de Milán y Alcalde de Casa y Corte, mientras otro tío, José Íñiguez de Abarca, colegial mayor de San Bartolomé de Salamanca, llegó a Vicario General del Obispado de Salamanca, Inquisidor de Córdoba y Prior de la Real Casa de Roncesvalles. Asimismo, un sobrino de Fernando Magallón, secretario de la embajada de París y de Parma, José Cubero Magallón, fue designado regidor de Daroca tras su retiro como cadete de infantería. 
Dentro de los regidores militares que no pertenecían a la oligarquía local, un rasgo los define primordialmente: su condición de forasteros ${ }^{6}$. La mayoría de ellos eran aragoneses nacidos en el mismo partido donde ejercieron su cargo, aunque también existe un buen número de originarios de poblaciones de partidos distintos. Tan sólo cuatro regidores militares nacieron en poblaciones peninsulares no aragonesas: Manuel Carrillo de Mendoza, nacido en Medinaceli (Soria), los navarros Marco Antonio Ureta Ayanz y Francisco Arno, y Andrés de Párraga Contreras, natural de Priego (Córdoba).

El caso más extraordinario es el de Jorge Domingo Traggia Aliprandi, italiano originario de Mezzana de Rabatón (Lombardía). Su oficio le fue concedido por deseo expreso de Felipe V el 9 de septiembre de 1728 sin mediar consulta previa por la Cámara, como pago a sus servicios en la Guerra de Sucesión. La Cámara presentó una queja al rey alegando su extranjería, procediendo a la petición de informes para conocer los antecedentes del sujeto. Uno de éstos fue emitido por el marqués de Risbourg, Capitán General de Cataluña, a cuyas órdenes se encontraba, ya que Traggia era en ese momento Gobernador de la Plaza de Balaguer, y a quién había servido en el sitio de Barcelona. Dicho informe fue positivo en todos los sentidos, como no podía ser menos, pero la frase final del mismo nos da una idea de cual era el estado de la pugna entre civilismo y militarismo, que por esos momentos se libraba sobre todo en los dominios de la antigua Corona de Aragón para controlar todos los ámbitos del poder: «... y que en sus Dominios y los de la Corona de Aragón, puede convenir que haya en las Ciudades Capitales algún Regidor de mayor seguridad para su Majestad y su Real servicio» ${ }^{7}$. Ante estos acontecimientos, el ayuntamiento desautorizó la toma de posesión de Traggia, pero tubo que plegarse a los deseos expresos del rey, que el 23 de diciembre le dispensó la extranjería y el 14 de enero del año siguiente expidió nuevo título de regidor.

Tanto estos regidores de origen forastero como el resto de los que acceden al poder desde fuera de la oligarquía local debieron el cargo a su carrera militar, que más tarde detallaremos, pero también a sus enlaces familiares. Buena parte pertenecían a familias con amplios antecedentes militares, como los casos de Manuel Omul-Ryan y Marco Antonio Ureta Ayanz, regidores de Huesca. Los dos abuelos de Omul-Ryan habían sido militares (el paterno Sargento Mayor de Arén y el materno capitán en la misma Plaza), lo mismo que su padre (Ayudante de la Plaza de Arén) y uno de sus hermanos (Guardia de Corps). Por su parte, Marco Antonio Ureta era sobrino de varios militares repartidos por los territorios de la Monarquía y con altas graduaciones: Mariscal de Campo en Perú, capitán en Flandes, capitán en Cataluña y Mariscal de Campo en Larache.

Otro grupo de regidores militares ajenos a la oligarquía podían incluir en su $\mathrm{cu}$ rriculum, además de esta condición, servicios familiares en diferentes instancias de la administración: Alonso de Villalpando, conde de Torresecas y marqués de la Com-

6. Existieron un total de 23 regidores no nacidos en el municipio donde ejercieron (un $24,5 \%$ ).

7. A.H.N., Consejos, leg. 18.095. El Excmo Sr. Marqués de Risbourg a Don Antonio Bescansa, 2 de noviembre de 1728. 
puesta, era sobrino de José Rodrigo Villalpando, de larga y extensa carrera ${ }^{8}$. También otros menos destacados tenían, o habían tenido, parientes en las Audiencias, preferentemente la de Aragón, como fiscales u oidores. La configuración de este grupo de militares se completa con quienes tenían diversos familiares letrados vinculados a la universidad o a las asesorías municipales. También la Iglesia era fuente de buenas referencias para este grupo de regidores sin relación con el gobierno local, como la familia de Bernabé de Roa, cuyos tíos ocupaban varias instituciones eclesiásticas de Aragón: uno Dignidad de la Catedral de Tarazona, otro Deán de la de Albarracín y un tercero como canónigo de la de Teruel.

\section{c) Posición económica}

La situación económica de los regidores militares está condicionada por esa división que hemos establecido entre los que provenían de la oligarquía local y los que se incorporaban a ella, bien desde fuera del municipio o desde el interior del mismo. Los que ya eran miembros del grupo de poder local mantenían una clara vinculación con la posesión de tierras, requisito que siempre fue considerado como indispensable para acceder al cargo de regidor. Por ello, el oficio militar fue, en ocasiones, estimado como insuficiente si no se poseía un patrimonio en bienes raíces competente para mantener el decoro del oficio, y su propia persona y familia ${ }^{9}$. Pero existía una estratifícación muy variopinta entre los regidores militares que disfrutaban de propiedades rústicas: nobles titulados de Zaragoza y otras localidades, que gozaban de dominios feudales más o menos extensos, dueños de pequeños señoríos, pero sin título de nobleza ${ }^{10}$, propietarios de mayorazgos o simples hacendados.

Sin embargo, no podemos perder el referente de que el ejército fue un buen método para acceder a otras cotas de poder, en este caso el municipal. Ya mencionamos la presencia de regidores militares forasteros, cuyo arraigo en la localidad solía ser escaso y por ello fuente de numerosas quejas por parte de la oligarquía local ante su entrada". Pero también hemos localizado individuos que por sus antecedentes familiares estaban ligados a actividades no agrarias, lo que les valió informes negativos

8. Lugarteniente del Justicia de Aragón en 1699, ejerció como fiscal en la primera planta de la Audiencia borbónica de Aragón en 1711 y dos años más tarde destinado a la embajada de París, de donde volvió en 1717 para ocupar la Secretaría de Gracia y Justicia.

9. Sobre Lorenzo Marqués, que pretendía un regimiento en su ciudad natal de Alcañiz tras haber ejercido como sargento de las Guardias Españolas, decía la Audiencia: «en ella no se le sabe más bienes que el de su nacimiento y bautismo, ni pariente, que no sean de igual pobreza, y extracción, ... no puede ser este oficio premio para su retiro, si antes no se establece con algún Patrimonio competente, para vivir y habitar en aquella Ciudad, donde ha de residir», A.H.N., Consejos, leg. 18.072. La Audiencia a 17 de julio de 1767.

10. En Zaragoza, Joaquín Ignacio Escala, señor de Finestras y cuadra de Espluga; en Benabarre, Francisco Aguirre y Escala, señor de Alcanar y Caladrones; en Huesca, José Castilla Cavero, señor de Ortilla, y Justo de Urriés, señor de Nisano.

11. Fermín Ram de Viu debió acreditar sus posesiones en Calanda, que ascendían a la considerable cifra de más de 10.000 libras, a través de la consulta del Catastro de dicha localidad para poder acceder al ayuntamiento de Alcañiz, A.H.N., Consejos, leg. 18.073. Informe de la Audiencia, 7 de octubre de 1805. 
por parte de los ayuntamientos. La mayoría de ellos provenían de familias comerciantes, aunque los había con antecedentes en oficios artesanos ${ }^{12}$. Igualmente, algún regidor procedía de familias ligadas a establecimientos industriales de mayor fuste, como Pedro Campillo, regidor de Borja, cuyo abuelo había establecido una fábrica de escopetas de caza en Villafeliche que en tiempos de Felipe V pasó a propiedad del Estado, pero siguiendo su familia como administradora de la misma.

\subsection{Carrera militar}

El análisis de la carrera militar de estos regidores también nos indica que se trataba de un grupo heterogéneo. En primer lugar, unos ya habían sido retirados del servicio (aproximadamente dos tercios de este colectivo), mientras otros seguían en activo, a pesar de que ello comportaba el alejamiento físico del cargo, lo que fue fuente de queja por parte de los municipios que, en algunos casos, cuando el sujeto lograba la anuencia real, debía seguir pagando el salario, o, aunque así no fuese, veía como el trabajo de los demás se acrecentaba al decrecer su número.

Buena parte de los retirados lo fueron tras las campañas de la Guerra de Sucesión ya que su carrera militar estaba muy supeditada a este momento concreto por lo que volvieron a sus lugares de origen, o al de su mujer en el caso de algunos regidores que veían más necesario cuidar del patrimonio conyugal. Las razones del retiro venían motivadas por la extracción social de estos individuos que debían volver a su localidad natal a cuidar de su familia y hacienda al ser, en muchos casos, el hijo primogénito, lo cual afectó no sólo a los miembros de la baja nobleza sino también a la alta nobleza ${ }^{13}$. Pero también se podía deber a los avatares del servicio: enfermedad o heridas de guerra. En algunas ocasiones el retiro suponía la agregación a las diferentes plazas militares aragonesas, fundamentalmente Zaragoza, pero también Monzón (Manuel Omul-Ryan, regidor de Huesca), e incluso de fuera de Aragón (Fernando París, regidor de Sos, agregado a la de Pamplona).

La graduación militar, en el momento de ser designados regidores, no era muy elevada por término general, pues predominan los de capitán, cadete y teniente. Sin embargo, algunos alcanzaron la de coronel ${ }^{14}$, o de teniente coronel ${ }^{15}$. Sólo en el caso

12. En Borja, Diego Cenón Navarro contaba entre sus abuelos un maestro alarife; en Tarazona, Félix Asensio tenía antecedentes en el oficio de sastre y Manuel Navarro Iturren entre los zapateros.

13. Entre los primeros, Lorenzo Lázaro San Gil volvió en 1719 a cuidar de su hacienda y familia (madre y once hermanos) en Borja, tras la muerte de su padre, regidor de la misma; entre los segundos, Gregorio Ignacio de la Sierra, hijo y sucesor del título de barón de Letosa, cuyo padre le cedió la plaza de regidor de Zaragoza.

14. De los cuatro que lo hicieron, tres fueron regidores de Zaragoza y dos pertenecían a la alta nobleza (el marqués de Villasegura y Rafael Salabert), mientras el cuarto sólo alcanzó esta distinción entre los cuerpos de milicias (Pedro Foradada en Fraga), aunque era militar de profesión.

15. También todos en Zaragoza y miembros de la alta nobleza, excepto Francisco Domenech Foradada, regidor de Huesca (y anteriormente de Fraga durante un trienio), igualmente en los cuerpos de milicias. 
de la alta nobleza, especialmente la que ocupó las regidurías de la capital, pudieron ascender a los más altos grados: Miguel López Fernández Heredia (Teniente General de Caballería), Antonio María Salabert, marqués de Valdeolmos, y José Fonsdeviela Ondeano, marqués de la Torre (brigadieres), y el conde de Bureta (Mariscal de Campo).

Por lo que respecta a los cuerpos en los que servían, el más frecuente fue el de caballería, como correspondía a su extracción social, seguido del de infantería; pero también fue habitual el servicio en el de Guardias (todos, a excepción de Benito María de Ciria Beteta, regidor de Calatayud, que lo hizo en las Valonas, en las Españolas) y en el de Dragones. El resto de cuerpos sólo fue ocupado por algún regidor esporádico: el de la Armada (Miguel Valero de la Hoz, regidor de Albarracín), el de Granaderos (Carlos Macdonell, regidor de Barbastro), el de Ingenieros (Rafael Firmat, regidor de Daroca), el de Artillería (José de Chueca y Joaquín Ignacio Escala, regidores de Zaragoza) y el de Carabineros (Estanislao García de Vera, regidor de Zaragoza); incluso un regidor (Fermín Ram de Viu, de Alcañiz) sirvió en la Orden de Malta, como caballero de San Juan que era.

Aparte de los servicios militares en campaña, que abarcan todos los que desarrolló la monarquía desde la Guerra de Sucesión a la Guerra de la Convención, nos interesa destacar que algunos pretendientes podían alegar méritos relacionados con la administración del ejército. Estrictamente militares eran los que habían ocupado la gobernación de plazas ${ }^{16}$, corregimientos militares ${ }^{17} \mathrm{o}$ alcaldías mayores de Indias ${ }^{18}$; pero también otras de carácter económico dentro de la milicia ${ }^{19}$ o fuera de ella (administración de Salinas, de Aduanas o de Correos).

Como ya hemos venido comprobando, la carrera militar era más importante entre los regidores de la capital del Reino, lo que queda corroborado analizando la de los que siguieron en activo. Sólo tres regidores no zaragozanos ocuparon cargos posteriores a su nombramiento municipal: Miguel Valero, de Albarracín, que fue Gobernador del Grao de Valencia en 1817, Mariano Lobera, de Calatayud, empleado en el corregimiento de Castellón entre 1785 y 1789 , y Justo de Urriés que tuvo ese mismo cargo en Daroca (1758-1765) y Calatayud (1765-1768).

Los regidores de la capital pasaban sobre todo a corregimientos militares o de capa y espada (el conde de Bureta en Gerona de 1731 a 1733; el barón de Letosa en

16. El anteriormente citado Francisco Domenech Foradada había ocupado los cargos de Gobernador de Mequinenza y Comandante del castillo de Lérida durante la Guerra de Sucesión; Antonio María Salabert, antes de una regiduría de Zaragoza, desempeñó el puesto de Gobernador y Corregidor de Zamora, y José Tomás Garcés Marcilla el de Gobernador de San Mateo (Castellón) de la Orden de Montesa antes de llegar al ayuntamiento de Teruel, aunque siguió ejerciendo el cargo posteriormente; más destacado era el cargo de Miguel López Fernández de Heredia, Comandante General de Canarias, previamente a su ingreso en el concejo zaragozano.

17. Francisco Aguirre Escala, regidor de Benabarre, que lo había sido de Chinchilla.

18. Francisco Javier Solanot volvió a ocupar una regiduría en la capital desde las alcaldías de Cuicatlán y Papalotipac en Nueva España.

19. Como el de Comisario Ordenador que ocuparon los regidores de Zaragoza José de Chueca, José de Fonsdeviela y Marcos Mayoral. 
Logroño de 1758 a 1763 y de Plasencia de 1770 a 1773; Juan García de Vera en Daroca de 1756 a 1757; y José Joaquín de Traggia en Cinco Villas de 1787 a 1792), a Intendencias de ejército (José Fonsdeviela lo fue sucesivamente de las de Sicilia y Nápoles, de Valencia, de Mallorca y de Extremadura), o unidas a los corregimientos (Francisco Javier Solanot que primero ocupó la de León y más tarde el corregimiento de Badajoz y la Intendencia de Extremadura); pero también a gobernaciones militares (el propio conde de Bureta en Rosas y su hijo María José Marín en Siracusa), o incluso cargos en el extranjero, como Felipe Fonsdeviela que era a su muerte en $1785 \mathrm{Mi}$ nistro Plenipotenciario en la Corte de Rusia.

La carrera más brillante quizá fue la de José Torrero Marzo, conde de la Rosa, que la comenzó en el corregimiento de Teruel (1733); tras un espacio dilatado de tiempo en que sirvió al Infante Cardenal, fue nombrado embajador en Portugal en 1760 y consejero de capa y espada del Consejo de Indias tres años más tarde, aunque murió casi sin poder ejercer el cargo. 